\title{
Original Article: A Clinical Study of Pattern of Microbiological Colonization of Endotracheal Tube Aspirate on Mechanically Ventilated Patients
}

\author{
Kiran Tandia (MD) ${ }^{1}$, J. L. Wadhwani (MD) ${ }^{2}$, Manuj Sharma (DM) ${ }^{3}$ \\ ${ }^{1}$ Resident Medical Officer Department of Medicine Gandhi Medical College Bhopal, India \\ ${ }^{2}$ Associate Professor Department of Medicine Gandhi Medical College Bhopal, India \\ ${ }^{3}$ Assistant Professor Department of Medicine Gandhi Medical College Bhopal, India
}

\begin{abstract}
Introduction: Nosocomial infection is a critical issue among intubated patients which is responsible for significant morbidity and mortality of these patients. One of the most important types of this infection is pneumoia which commonly occurs in relation to endotracheal tube and mechanical ventilation named ventilator associated pneumonia. .study presents the most common microorganisms colonized from endotracheal tube of hospitalized patients in our setup and their antibiotic sensitivity pattern. Methods: 100 patients who are admitted in emergency medical ward of Hamidia Hospital and then subsequently intubated and assisted by mechanical ventilation due to insufficient self ventilation are taken for study. Samples for microbiological cultures were taken after 48 hours of intubation by mucous extractor under all aseptic precautions and then sent to Microbiology laboratory for culture and sensitivity test. Results: our study showed pseudmonas is most common organism isolated followed by klebsiella and staphylococcus aureus and most of the organism were sensitive to piperacillin-tazobactam and meropenem. Discussion: prevalence of Ventilator associated pneumonia in our setup was bserved as $25 \%$.The bacteriology of positive sputum culture is caused predominantly by psedomonas aeruginosa in $17 \%$ followed by klebsiella $6 \%$ and staphyloccocus aureus $2 \%$.
\end{abstract}

Keywords: ventilator associated pneumonia, clinical pulmonary infection score, tracheal secretion, endotracheal tube, antibiotic sensitivity

\section{Introduction}

Infections are the most important and leading cause of mortality and morbidity among the patients admitted in ICU. Nosocomial infections is a critical issue among intubated patients which is responsible for significant morbidity and mortality of these patient" One of the most important types of this infection is pneumonia which commonly occurs in relation to the endotracheal tube and mechanical ventilation named ventilator associated pneumonia. Patients with mechanical ventilation have an increased risk for respiratory tract infection because the tube which has been inserted in trachea reduces the clearance of bacteria and increases the leakage of secretion around the cuff of tube and disable the cilliary tract by damaging it. Nosocomial infection is a frustrating and budget consuming issue and due to increased time of hospitalization it imposes a heavy burden on health care resource.

\section{Aims and Objectives}

- To study types of microorganisms in intubated patients [ $>48$ hour] in emergency medical ward of Hamidia Hospital Bhopal.

- To study microbiological antibiotic sensitivity in intubated patients $[>48$ hour] in emergency medical ward.

- To propose a proper empirical antibiotic therapy in intubated patients according to prevalence of microbiological organisms in emergency medical ward.

\section{Material and Method}

It was Observational Cross- Sectional study of 1 year period. 100 patients who were admitted in emergency medical ward of Hamidia Hospital Bhopal and then subsequently intubated and assisted by mechanical ventilation due to insufficient self ventilation were taken for study.Samples for microbiological cultures were taken after 48 hour of intubation by mucous extractor under all aseptic technique and then sent to Microbiology laboratory for culture and antibotic sensitivity test.

\section{Inclusion Criteria}

Patients intubated for $>48$ hour in emergency medical ward due to insufficient self ventilation.

\section{Exclusion Criteria}

- Patients who already had any respiratory tract infection or any other disease related to respiratory system.

- Patients who are intubated for $<48$ hour.

A total of 100 patients who were kept on mechanical ventilator were randomly selected.. All necessary measures were taken for prevention of hospital-acquired infections.

Ventilator associated pneumonia was diagnosed on clinical grounds based on the modified Clinical Pulmonary Infection Score (CPIS) system originally developed by Pugin and others, giving 0-2 points each for fever, leukocyte count, oxygenation status, quantity and purulence of tracheal secretions, type of radiographic abnormality and result of sputum culture and Gram stain. The Ventilator associated pneumonia group was classified into two groups, early-onset type (within 48-96 h) and late-onset type ( $>96 \mathrm{~h}$ ). Once the 


\section{International Journal of Science and Research (IJSR) \\ ISSN (Online): 2319-7064}

Index Copernicus Value (2013): 6.14 | Impact Factor (2014): 5.611

clinical suspicion was established, empirical antibiotic therapy was initiated based on guidelines prescribed by the American Thoracic Society. Patients were routinely screened by arterial blood gas (ABG) analysis every 12 hourly and appropriate steps were taken to correct any change.

\section{Clinical Pulmonary Infection Score (CPIS)}

Clinical Pulmonary Infection Score (CPIS)

\begin{tabular}{|lr|}
\hline Parameter & Score \\
Temperature $(* C)$ & 0 \\
$>36.5$ and $<38.4$ & 1 \\
$>338.5$ and $<38.9$ & 2 \\
$>39.0$ or $<36.5$ & \\
White Blood Cell Count & 0 \\
$>4,000$ and $<11,000$ & 1 \\
$<4,000$ or $>11,000$ & 2 \\
$<4,000$ or $>11,000$ \& band forms $>50 \%$ & \\
Tracheal secretions & \\
Scanty & 1 \\
Purulent & 2 \\
Pao2/Fio2 & \\
$>240$, ARDS or pulmonary contusion & 0 \\
$<240$ and no ARDS & 2 \\
Chest Radiograph & \\
No infiltrate & 0 \\
Diffuse or patchy infiltrate & 1 \\
$\bullet$ Localised infiltrate & \\
\hline
\end{tabular}

\section{Result}

The most common microorganism isolated from endotracheal tube in our set up is pseudomonas aeruginosa followed by klebsiella and s.aureus. Moreover, most of the microorganisms were sensitive to and pipracillinTazobactam and meropenem.

\section{Discussion}

Prevalence of ventilator associated pneumonia in our setup is observed as $25 \%$. The bacteriology of positive sputum culture is caused predominantly by pseudomonas aeruginosa in $17 \%$ followed by klebsiella $6 \%$ and s.aureus $2 \%$.

It is analysed in our study that those requiring prolonged ventilator support had a significantly higher incidence of ventilator assoiated pneumonia ( $P$-value, 0.003$)$ of the 25 patients who developed ventilator assoiated pneumonia, 9 patients developed early-onset $(14.8 \%)$ ventilator assoiated pneumonia and 16 patients developed the late-onset type $(41.0 \%)$.In our study it is also found that purulent secretions associated with positive sputum culture ( $\mathrm{p}$ value $<0.001$ ).

In the study of our set up the incidence of ventilator assoiated pneumonia is $25 \%$ supported by Kalanuri et $\mathrm{al}^{[1,2]}$. Kalanuri et $\mathrm{al}^{[2]]}$ found ventilator assoiated pneumonia (VAP) estimated to occur in $9-27 \%$ of all mechanically ventilated patients, with the highest risk being early in the course of hospitalization.
The study also shows that patients with longer duration of hospital stay,had more chances of development of ventilator associated pneumonia ( $\mathrm{p}$ value, 0.03 ]

The study by Simoni et al ${ }^{[3,4]}$. showed that $100 \%$ of samples from airway prosthesis are positive in culture; however, other studies have reported a positive culture rate between $0 \%$ and $33 \%$ in obtained samples from airway tubes. Cardinosa et al. have reported a positive culture result in $89 \%$ of their samples. The variation could be explained by the technique of intubation, clinical and individual characteristics of study population, colonization during intubation or lack of sufficient precautions for intubation due to the high work load in an emergency setting .

In our study, Gram negative bacteria were the most common isolated organisms including Pseudomonas aeruginosa and Klebsiella which is in the same line with the study by Nardi

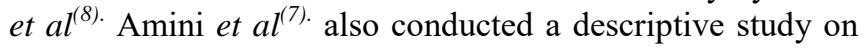
distribution of isolated microorganisms from tracheal tube of ICU patients declaring that S.aureus (23.6\%),Klebsiella species (23.3\%), Acintobacter species. (20.7\%), P.aeroginosa (18.2\%), E.coli (7.7\%), and Enterobacter species, were the most common isolates ${ }^{[8] .}$ Our study as well as the other ones in similar settings confirm that $P$. aeruginosa, klebsiella and $S$. aureus are among the most 3 prevalent isolated organism from endotracheal tube aspirate.

In contrast, Rello et $a l^{[5,6]}$ in their study have demonstrated that Pseudomonas aeruginosa is the most common causative organism for infection of Endotracheal tube and this could be the result of large number of patients with chronic obstructive pulmonary disease (COPD) and long time of intubation and even a previous history of antibiotic therapy It should be noted that although there are minor variations in relative frequency of obtained organisms, different studies have reported varying sources of isolations as in our study we did report colonization of organisms into the Endotracheal tube while other studies may have considered Endotracheal tube related infections in their study analysis.

\section{Conclusion}

Our study shows pseudomonas is the most common microorganism isolated from endotracheal tube in our set up. Moreover, most of the microorganisms were sensitive to and pipracillin-Tazobactam and meropenem. Though our study comprise 100 number of patients, for the result of the study to be applicable to general population and in our setup we require furthur studies with larger number of study subjects.

\section{Acknowledgment}

We sincerely thanks to all members of Emergency Medical ward of department of medicine of Gandhi Medical college and Hamidia Hospital Bhopal.

\section{References}

[1] Vincent JL, Bihari DJ, Suter PM, Bruining HA, White J, Nicolas-Chanoin MH, Wolff M, Spencer RC, Hemmer 


\section{International Journal of Science and Research (IJSR) \\ ISSN (Online): 2319-7064}

Index Copernicus Value (2013): 6.14 | Impact Factor (2014): 5.611

M: The prevalence of nosocomial infection in intensive care units in Europe. JAMA 1995, 274:639644.

[2] Kalanuria et al. Atul Ashok Kalanuria1, Wendy Zai2 ${ }^{*}$ and Marek Mirski, licensee Springer-Verlag Berlin Heidelberg and BioMed Central.

[3] Hina gadani, Arun Vyas,Akhya kumar Kar, A study of ventilator associated pneumonia: incidence, outcome, risk factors and measures to be taken for prevention.

[4] Pugin J, Auckenthaler R, Mili N, Janssens JP, Lew PD, Suter PM: Diagnosis of ventilator associated pneumonia by bacteriologic analysis of bronchoscopic and non bronchoscopic "blind" bronchoalveolar lavage fluid.

[5] Simoni P, Wiatrakm Bj. Microbiology of stents in laryngotracheal reconstruction.

[6] Cunnion ,K.M.,D.J. weber, W.E. Broadhead, L.C.Hanson , C.F. Pieper and W Cardenosa Cendrero JA, Sole-Violan J, Bordes Benitez A ,Noguera Catalan J, Arroyo Fernandez J, saavendra Santana $p$ et al. Role of different routs of tracheal colonization in the development of pneumonia in patients receiving mechanical ventilation.

[7] Amini M Javanmard A, Davati A, Azimi G. Bacterial colonization in tracheal tubes of ICU patients. Iranian Journal of Pathology. 2009;4:123-127.

[8] Johnson WG, Pierce AK, Sanford JP, Thomas GD: Nosocomial respiratory infections with gram negative bacilli. The significance of colonization of the respiratory tract Oncag O Cokmez B Aydemir S, Balcioglu T.
Table 1: Distribution of Cases According to Duration of Stay

\begin{tabular}{|c|c|c|c|}
\hline Duration of stay & Culture Positive & Negative & Total \\
\hline \multirow{2}{*}{$<4$ days } & 9 & 52 & 61 \\
\cline { 2 - 4 } & $14.8 \%$ & $85.2 \%$ & $100.0 \%$ \\
\hline \multirow{2}{*}{$>4$ days } & 16 & 23 & 39 \\
\cline { 2 - 4 } & $41.0 \%$ & $59.0 \%$ & $100.0 \%$ \\
\hline \multirow{2}{*}{ Total } & $\mathbf{2 5}$ & $\mathbf{7 5}$ & $\mathbf{1 0 0}$ \\
\cline { 2 - 4 } & $\mathbf{2 5 . 0 \%}$ & $\mathbf{7 5 . 0 \%}$ & $\mathbf{1 0 0 . 0 \%}$ \\
\hline
\end{tabular}

$\mathrm{P}=0.003$ [significant, Longer stay more VAP

Table 2: Distribution of Cases According to Type of Tracheal Secretions

\begin{tabular}{|c|c|c|c|}
\hline Tracheal Secretions Type & Culture Positive & Negative & Total \\
\hline \multirow{2}{*}{ purulent } & 9 & 0 & 9 \\
\cline { 2 - 4 } & $100.0 \%$ & $0.0 \%$ & $100.0 \%$ \\
\hline \multirow{2}{*}{ scanty } & 16 & 75 & 91 \\
\cline { 2 - 4 } & $17.6 \%$ & $82.4 \%$ & $100.0 \%$ \\
\hline \multirow{2}{*}{ Total } & 25 & 75 & 100 \\
\cline { 2 - 4 } & $25.0 \%$ & $75.0 \%$ & $100.0 \%$ \\
\hline
\end{tabular}

$\mathrm{P}<0.001$ [ significant, all purulent tracheal secretions had vap

Table 3: Distribution of Cases According to Tracheal Aspirate Culture

\begin{tabular}{|c|c|c|}
\hline Tracheal aspirate culture & Number of cases & \% of cases \\
\hline Klebsiella & 4 & 4.0 \\
\hline Pseudomonas & 17 & 17.0 \\
\hline S. aureus & 2 & 2.0 \\
\hline Negative & 75 & 75.0 \\
\hline Total & $\mathbf{1 0 0}$ & $\mathbf{1 0 0 . 0}$ \\
\hline
\end{tabular}

Table 4: Distribution of Case According to Tracheal Aspirate Culture and Their Antibiotic Sensitivity Pattern

\begin{tabular}{|c|c|c|c|c|c|c|}
\hline Antibiotics & $\begin{array}{c}\text { Klebsiella } \\
{[4]}\end{array}$ & $\begin{array}{c}\% \text { Klebsiella } \\
\text { sensitivity }\end{array}$ & $\begin{array}{c}\text { Pseudomonas } \\
{[17]}\end{array}$ & $\begin{array}{c}\% \\
\text { Pseudomonas } \\
\text { sensitivity }\end{array}$ & $\begin{array}{c}\text { S Aureus } \\
{[4]}\end{array}$ & $\begin{array}{c}\% \text { S Aureus } \\
\text { sensitivity }\end{array}$ \\
\hline Ceftrioxone & 1 & 16.6 & 0 & 0.0 & 0 & 0.0 \\
\hline Piperaciliin-Tazobactam & 6 & 100.0 & 15 & 88.2 & 0 & 0.0 \\
\hline Meropenem & 3 & 50.0 & 11 & 64.7 & 0 & 0.0 \\
\hline
\end{tabular}

